\title{
The Influence of Leader Prestige on Subordinates' Job Attitude and Behavior: mediation and moderation effects
}

\section{$\mathrm{Li} \mathrm{He}$}

Faculty of Politics and Public Management, Guangdong University of Foreign Studies, 510006, China

\begin{abstract}
To explore the problem of leader prestige Chinese is familiar with, an empirical study was conducted. Base on the results of a sample of full-time employees in enterprises, the present study analyzed the effects of leader prestige on subordinates' job attitude and behavior. The study proves that leader prestige can improve leader trust and organization identification of subordinates. Leader prestige can give birth to positive effects on leader trust, and then improve organizational identification of subordinates. Leader prestige has positive effects on job satisfaction and job engagement by the full mediator of organizational identification and leader trust. Leader's power and leader-member exchange can moderate the positive relationship between leader prestige and subordinates' organizational identification, but cannot moderate the relationship between leader prestige and leader trust.
\end{abstract}

Keywords. leader prestige; leader trust; organizational identification; job satisfaction; job engagement; leader's power; leader-member exchange

\section{Introduction}

For a long time, the prestige is an important index to inspect the leaders/administrative cadres in China, and also an important basis to know whether the people advocate their leaders/managers. An efficient leadership, namely the leadership prestige of manager, depends on the influence on the subordinates. It determines the comprehensive management efficiency of the manager. In Chinese society, the leadership in consistency with Chinese cultural features has a great effect, but the one not in consistency with that has a bad effect. (Jingli Fan, Boxun, Zheng, 2000). Currently, the foreign scholars have proposed multiple leadership theories similar to Chinese concept of leadership prestige. While domestically, some leadership concept and leadership theories with Chinese cultural features are still in the theoretical stage. Therefore, it is a traditional but advanced subject to conduct a research on leadership prestige of Chinese managers, which integrates the latest foreign research results of leadership theory and long-term practice of domestic leadership culture.

\section{Research theory and research hypothesis}

\subsection{Leadership prestige in Chinese culture—existed as an influence}


As the word "prestige" was proposed in Chinese cultural background, there's no equivalent concept in foreign countries. Prestige includes the meaning of authority and credit. Prestige is potential authority and influence of people's personality, an influence without power, as well as a sincere compliment, respect and trust to the person in a certain position (Qi Zheng, 2008). In the influence process of personal prestige, the leader uses power, knowledge, talent and personal charm to influence and manage the managed individual or group to realize the organizational goal.

As for the effect of culture on leadership theory, Boxun Zheng, et al. (2003) thought the connotation, demeanor and practical behavior of leader would be affected by culture. (Boxun Zheng, et al. 2003). Currently, there's no theory about leadership prestige in western countries that can be directly quoted because there is still some difference in the research perspective of leadership theory issues in Chinese and western culture. The existing leadership about leadership prestige in Chinese culture mainly includes authentic leadership, charming leadership and paternalistic leadership. While those concepts are about a leadership manner, a little different from the concept "influence" highlighted in leadership prestige.

In the concept of authentic leadership with closest meaning to "prestige", the words Authentic Leadership also includes the meaning "prestige" in Chinese culture. Luthans et al. (2003) thought the authentic leadership refers to a process in which the positive psychological ability of leader and highly developed organizational context are integrated to function. The "authentic leadership" or "prestige leadership" mainly refers to the manner or process of leadership, but the "leadership prestige" in China mainly refers to leader's personal prestige and influence, indicating it's just a personal feature for the leader.

In the discussion of leadership prestige among domestic scholars, Suhua Qian, Weiguo Min (2002) thought the flexible management method must be strengthened to establish leadership prestige. Apart from power and position, the prestige of manager shall also include manager's personality, quality, knowledge, ability and other factors. Qi Zheng (2008) thought there are forced and voluntary influence, the former referring to power, the latter to prestige. But having power does not necessarily mean to have prestige at the same time; The source of prestige includes morality, knowledge, talent, integrity, honesty, etc.. From what is mentioned above, the "leadership prestige" is construed by Chinese as the comprehensive influence of manager himself in leadership or management. Considering all the above analysis, this research says the leadership prestige in Chinese culture can be defined as: an influence, without power, produced by leader's own factors and behaviors and, with which the leader will be capable of winning the advocacy and support from subordinates.

\subsection{Research hypothesis}

\subsubsection{Effect of leadership prestige on satisfaction and working investment of subordinate}

Avolio et al. (2004) made a research on the influence mechanism of authentic leadership and subordinate attitude and behavior. It is found the authentic leadership improves the expectation, trust, positive emotion and other psychological statuses through identity of subordinate with leader's personal and social status, and further influences the organizational commitment, working investment and working satisfaction of employees. In relevant discussion, Qi Zheng (2008), Suhua Qian, Weiguo Min (2002) et al. had made analysis on the attraction and influence of leadership prestige on psychology and behavior of the subordinate and the people. So this research proposes the following hypothesis:

Hypothesis 1: The leadership prestige has a significant positive effect on working satisfaction of subordinate.

Hypothesis 2: The leadership prestige has a significant positive effect on working investment of subordinate. 


\subsubsection{Mediation effect of subordinate trust for leader}

It is found from the research of paternalistic leadership made by Jingli Fan and Boxun Zheng (2000) that moral leadership and merciful leadership have a significant positive effect on the trust of subordinate for its superior, while the authoritative leadership has a significant negative effect. Dirks and Ferrin (2002) proposed a set of theoretical framework "leadership behavior--superior trust--employee response"; If the subordinate develops trust for its superior, they will tend to establish a mutual caring relation, and further conduct positive working behavior. In Chinese traditional culture, the employees are easier to develop trust and feeling of dependence on the leader with authority or prestige, and probably show more positive working responses. Based on the above analysis, this research proposes the following hypothesis:

Hypothesis 3: The subordinate trust for leader has a mediation effect on leadership prestige and working satisfaction.

Hypothesis 4: The subordinate trust for leader has a mediation effect on leadership prestige and working investment.

\subsubsection{Mediation effect of organizational identity of subordinate}

The empirical research of Epitropaki et al. (2005) said the innovative leader will have a positive effect on the organization identity of the employee. This is because the organizational identity of organizational members is to meet its identity with organizational goal and meaning, and the leader is a conveyor of organizational meaning. So the innovative leader will induce a sense of organizational identity for the employee. Bergami et al. (2000) made a research and found the organizational identity can significant improve the organizational citizenship behavior, organizational commitment and contribution and professional dedication for the employee. Li He, Wenquan Ling (2008) also proved the organizational identity has a significant promotive effect on working investment of the employee through empirical research. So this research proposes the following hypothesis:

Hypothesis 5: The organizational identity has a mediation effect on leadership prestige and working satisfaction.

Hypothesis 6: The organizational identity has a mediation effect on leadership prestige and working investment.

\subsubsection{Regulating function of leadership}

In an organization, if the superior has many official powers (such as power of bonus, punishment and resources allocation), its subordinate will develop a sense of dependence on it, and this is just the source of subordinate's feeling for the power of its superior. And Jian Zhang (2009) also thought the power of leader functions mainly under the influence of prestige. A qualified leader needs to organically integrate the power and prestige to guide, stimulate and restrain its subordinate and common people. So this research proposes the following hypothesis:

Hypothesis 7: The leader power can strengthen the relation of manager's leadership prestige and subordinate's organizational identity.

Hypothesis 8: The leader power can strengthen the relation of manager's leadership prestige and subordinate's trust for leader.

\subsubsection{Regulating function of leader-member commutation relation}

As for the leader and subordinate in good leader-member commutation relation, the subordinate will positively keep in consistency with its superior and organization, as well as show an attitude and behavior compliant with the requirement of organization and superior to maintain and develop a good relation with its superior. While Liden \& Maslyn (1998) also found from their research that 
a high-quality leader-member relation has an important effect on the working behavior and working attitude of subordinate. Sufficient mutual trust and dependence between the leader and subordinate will also induce organizational identity of the subordinate. So this research proposes the following hypothesis:

Hypothesis 9: The leader-member commutation relation can strengthen the relation of manager's leadership prestige and subordinate's organizational identity.

Hypothesis 10: The leader-member commutation relation can strengthen the relation of manager's leadership prestige and subordinate's trust for leader.

\section{Research method and research process}

\subsection{Investigation process}

The investigated objectives selected in this research are a certain number of enterprises in different types and the official members therein, and they cover multiple enterprises or groups with service time above 1 year. There's just one direct superior at present. The investigation was conducted for current students in MBA class in Pearl River Delta region and the enterprise employees in the company's training course. There were 600 questionnaires issued in the investigation, where 408 valid questionnaires were returned. The rate of valid questionnaire returning was $68 \%$. The research data may have high stability because the number of valid samples, 408 , has exceeded the number of items in the longest questionnaire for 20 times.

\subsection{Investigated objective}

The enterprises where the investigated objectives are include general manufacture industry, hightech enterprise, service industry, etc.. Among which, in terms of the position, there were 216 people in regular position, accounting for $52.9 \% ; 217$ people in basic management position (such as director of department), accounting for $31.1 \%$; 58 people in intermediate management position, accounting for $14.2 \%$; 7 people in senior management position, accounting for $1.7 \%$;

\subsection{Research tool}

The questionnaire of leadership prestige is developed from the "leadership prestige" factors in organizational atmosphere scale made by Xiaofu Pan and Qiwen Qin (2007), including "the employee may feel the power of its leader"; "The leader has charisma and cohesive force" and the other 3 items, 5 in total. Through multiple researches made by Xiaofu Pan, et. al., those scale factors have desirable reliability and validity. The questionnaire involves an assessment made by a subordinate for its direct superior manager.

The questionnaire of subordinate's trust for leader prepared by Podsakoff et al. (1990) quoted by Farh et al. (1998) was used as the questionnaire of leader trust, where there are totally 4 items, such as "I am sure he (she) will treat me fairly" and "I firmly believe he (she) is honest".

The organizational identity questionnaire developed by Mael \& Ashforth (1992) was used as the organizational identity questionnaire. There is just one dimension and 6 items in the questionnaire, such as "I think the criticism from others against our company is a personal insult" and "The success of company is mine, too".

The 3rd scale in MOAQ developed by Cammann et al. (1983) was used as the questionnaire of working satisfaction, and the questions include "Generally, I am satisfied with my work", etc..

The questionnaire of working investment used totally 3 items in MOAQ prepared by Cammann et al. (1983), and the questions include "I am dedicated to my work" and "It is the most important for me to be dedicated to my work". Verified by Zongyou Wu and Boxun Zheng (2006), this questionnaire is proved to have a good reliability and validity. 
The questionnaire of leadership was adapted from the competent power scale of Ashforth (1997). We changed the superior for direct superior leader. There are 3 questions including "My leader has great power in the organization".

The LMX-7 questionnaire widely used in academic field was used as the questionnaire of leader-member commutation relation. Hui, Law and Chen (1999) translated this questionnaire into Chinese and used it in Chinese cultural background, including 7 questions in total, such as "My superior is very clear about my potential in the work" and "My superior will help me out of the troubles despite its own interest". The questionnaire was assessed by the subordinate.

All the above questionnaires in the investigation used Likert seven point scoring and required the respondent to determine the compliance degree of these assessments with the actual conditions according to the description of items. 1 signifies non-compliance, and 7 means good compliance.

\section{Research result}

\subsection{Analysis result of confirmatory factors}

This research uses latent variable path analysis method to verify the research hypothesis. The validity of questionnaire used in this research can be inspected as follows: In content validity, mature questionnaires were used as those seven questionnaires, and revised in the pretest process so as to ensure a fairly desirable content validity. Conduct analysis of confirmatory factors on the above six variables. In structural validity, we use absolute fit index ( $\left.\chi^{2} 、 \chi^{2} / \mathrm{df}, \mathrm{RMSEA}\right)$, relative fit index (CFI) and simple fit index (PNFI). As shown in the result of confirmatory analysis in Table 1, the fit index of basic model is relatively good, indicating seven questionnaires represent a relatively independent construct validity and have a good discrimination validity.

Table 1. Analysis result of confirmatory factors of concept discrimination $(\mathrm{N}=408)$

\begin{tabular}{llllllll}
\hline Model & $\chi^{\mathbf{2}}$ & df & $\chi^{\mathbf{2} / \mathbf{d f}}$ & RMSEA & CFI & TLI & PNFI \\
\hline Basic model & 1121.868 & 413. & 2.716. & .065 & .917 & .907 & .778 \\
Model 1 & 1311.704 & 419. & 3.131. & .072 & .896 & .885 & .770 \\
Model 2 & 2303.534 & 424. & 5.433. & .104 & .781 & .760 & .680 \\
Model 3 & 3256.188 & 429. & 7.590. & .127 & .671 & .643 & .590 \\
Model 4 & 3371.769 & 432. & 7.805. & .129 & .657 & .631 & .583 \\
Model 5 & 3239.509 & 434. & 7.464. & .126 & .673 & .650 & .599 \\
Model 6 & 3648.688 & 435. & 8.388. & .135 & .625 & .600 & .558 \\
\hline
\end{tabular}

Note: PRE stands for leadership prestige, POW for leadership, LMX for commutation relation, TRU for leader trust, ORG for organizational identity, SAT for working satisfaction, INV for working investment; + for factors integration.

Basic model: 7 factors: PRE, POW, LMX, TRU, ORG, SAT, INV

Model 1: 6 factors: PRE+POW, LMX, TRU, ORG, SAT, INV

Model 2: 5 factors: PRE+POW+LMX, TRU, ORG, SAT, INV

Model 3: 4 factors: PRE+POW+LMX, TRU+ORG, SAT, INV

Model 4: 3 factors: PRE+POW+LMX, TRU+ORG, SAT+INV

Model 5: 2 factors: PRE+POW+LMX+TRU+ORG, SAT+INV

Model 6: 1 factors: $\mathrm{PRE}+\mathrm{POW}+\mathrm{LMX}+\mathrm{TRU}+\mathrm{ORG}+\mathrm{SAT}+\mathrm{INV}$

\subsection{Descriptive statistical analysis on each variable}

Analyze the relevant matrix among each variable. The result in Table 2 shows the correlation of all the variables comply with the research expectation. As shown in Table 2, all the internal 
consistency factors of the scale used in this research are over 0.75 . Table 2 provides a necessary condition for the full-model construction and regulated variable analysis.

Table 2. Correlation of descriptive statistic result and variables ( $\mathrm{N}=408)$

\begin{tabular}{llllllllll}
\hline & $\mathrm{M}$ & $\mathrm{SD}$ & 1. & 2. & 3. & 4. & 5. & 6. & 7. \\
\hline 1. Leadership prestige & 5.583 & 1.163. & $(.940)$ & & & & & & \\
2. Leader trust & 5.479 & 1.236. & $.652^{* *}$ & $(.909)$ & & & & & \\
3. Organizational identity & 5.316 & .986 & $.388^{* *}$ & $.414^{* *}$ & $(.799)$ &. & & & \\
4. Working satisfaction & 5.305 & 1.125. & $.377^{* *}$ & $.505^{* *}$ & $.439^{* *}$ & $(.800)$ & & & \\
5. Working investment & 5.352 & 1.118. & $.333^{* *}$ & $.421^{* *}$ & $.473^{* *}$ & $.568^{* *}$ & $(.774)$ & & \\
6. Leadership & 5.396 & 1.134. & $.532^{* *}$ & $.501^{* *}$ & $.366^{* *}$ & $.373^{* *}$ & $.385^{* *}$ & $(.760)$ & \\
7. Commutation relation & 5.083 & 1.109. & $.582^{* *}$ & $.747^{* *}$ & $.482^{* *}$ & $.564^{* *}$ & $.463^{* *}$ & .533 & $(.920)$ \\
\hline
\end{tabular}

Note: ${ }^{* *}$ means $\mathrm{P}<0.01$, and * means $\mathrm{P}<0.05$, similarly hereinafter; The numbers in the brackets on the diagonal stands for Cronbach $\alpha$ factor of the scale.

\subsection{Hypothesis inspection}

\subsubsection{Inspection of action mechanism of leadership prestige}

This research uses latent variable path analysis method to verify the research hypothesis. The statistic software used is Amos 7.0.

In this research, the premises for mediation inspection are met according to related mode among 5 variables in Table 2. We construct a set of nested models for comparison to determine the relation of each variable. The Model 1 is the assumed original model that has set all the possible connection paths among 5 variables. As the paths from leader trust to working investment, from leadership prestige to working satisfaction and working investment are not significant, we use the gradual elimination method to respectively get Model 2 and Model 3 (as shown in Table 3). After eliminating these paths, the fitting of model to data is not significantly improved $(\Delta \chi 2)$, which indicates there's no direct path from leadership prestige to outcome variable. According to fit index and frugality principle, Model 3 is the optimal model, as shown in Figure 1.

Table 3. Fit index of each model $(\mathrm{N}=408)$

\begin{tabular}{lllllllll}
\hline Model & $\chi^{2}$ & df & $\chi^{2} / \mathbf{d f}$ & RMSEA & CFI & TLI & PNFI & $\Delta \chi^{2}(\Delta d f)$ \\
\hline Model 1 & 527.663 & 179. & 2.948. & .069 & .936 & .925 & .773 & - \\
Model 2 & 527.771 & 180. & 2.932. & .069 & .937 & .926 & .778 & $0.108(1)$ \\
Model 3 & 527.799 & 182. & 2.900. & .068 & .937 & .927 & .786 & $0.028(1)$ \\
\hline
\end{tabular}

Model 1: including the models of all the paths

Model 2: eliminate the path "leader trust $\rightarrow$ working investment";

Model 3: eliminate the path "leadership prestige $\rightarrow$ working satisfaction" and "leadership prestige $\rightarrow$ working investment";



Figure 1. Correction model of leadership prestige and its relevant variables (Model 3) 
As shown in the above research result: (1) The leadership prestige has a significant positive effect on both leadership prestige and leader trust $(\beta=0.67, \mathrm{P}<0.001 ; \beta=0.23, \mathrm{P}<0.01)$; (2) The leader trust has a significant effect on employee's working satisfaction $(\beta=0.39, \mathrm{P}<0.001)$; The employee's organizational identity has a significant positive effect on its working satisfaction and working influence $(\beta=0.40, \mathrm{P}<0.001 ; \beta=0.18, \mathrm{P}<0.01)$; (3) The employee's working satisfaction has a fairly strong positive effect on its working investment conditions $(\beta=0.60, \mathrm{P}<0.001)$; (4) The leader trust and organizational identity have a mediation effect between the leadership prestige and subordinate's working satisfaction and working investment. Considering all the above research results, the hypotheses 1-6 are all proved.

\subsubsection{Inspection of the effect of regulating variables}

To discuss whether there's a regulating effect between the leadership prestige and the organizational identity of subordinate, the independent variables and interactive effect variables are incorporated into the regression equation in order, for the purpose of predicting the effect on the organizational identity and other outcome variables. In the research, we conduct centralization treatment for each research variable, and calculate the interactive effect variables, as shown in Table 4.

Table 4. Regulating effect of leadership between leadership prestige and organizational identity of subordinate

\begin{tabular}{lll}
\hline Dependent variable $\rightarrow$ & \multicolumn{2}{l}{ Organizational identity of subordinate } \\
\hline $\begin{array}{l}\text { Explanatory variable } \downarrow \\
\text { Independent variables }\end{array}$ & $\mathrm{M}_{1}$ & $\mathrm{M}_{2}$ \\
$\quad$ Leadership prestige & $0.270^{* * *}$ & $0.320^{* * *}$ \\
$\quad$ Leadership & $0.222^{* * *}$ & $0.246^{* * *}$ \\
Interactive effect & & \\
$\quad$ Leadership prestige $\times$ Leadership & & $0.146^{* *}$ \\
$R^{2}$ & 0.186 & .203 \\
$\Delta R^{2}$ & $0.186^{* * *}$ & $0.017^{* *}$ \\
$F$ & $46.340^{* * *}$ & $34.341^{* * *}$ \\
\hline
\end{tabular}

As shown in Table 4, the leadership of superior has a significant regulating effect on the relation between leadership prestige and organizational identity of subordinate $\left(\mathrm{M}_{2}, \beta=0.146, p<\right.$ $0.01)$. So the hypothesis 7 is proved to be true. An analysis using the same method is made on the regulating effect of leadership between leadership prestige and organizational identity of subordinate, but its regulating effect is found to be not significant, so it is not indicated in a list. The hypothesis 8 is not proved.

Table 5. Regulating effect of leader-member commutation relation between leadership prestige and organizational identity of subordinate

\begin{tabular}{lll}
\hline Dependent variable $\rightarrow$ & \multicolumn{2}{l}{ Organizational identity of subordinate } \\
\hline Explanatory variable $\downarrow$ & $\mathrm{M}_{3}$ & $\mathrm{M}_{4}$ \\
Independent variables & & \\
$\quad$ Leadership prestige & $.164^{* *}$ & $.228^{* * *}$ \\
$\quad$ Leader-member commutation relation & $.387^{* * *}$ & $.383^{* * *}$ \\
Interactive effect & & $.127^{* *}$ \\
$\quad$ Leadership prestige $\times$ Leader-member commutation relation & & .262 \\
$R^{2}$ & .250 & .012 \\
$\Delta R^{2}$ & .250 & $47.851^{* * *}$ \\
$F$ & 67.415 & \\
\hline
\end{tabular}


Then, the same method is used to inspect the regulating effect of leader-member commutation relation between leadership prestige and organizational identity of subordinate. The result shows (see Table 5) a significant regulating effect of leader-member commutation relation between leadership prestige and organizational identity of subordinate $\left(\mathrm{M}_{4}, \beta=0.127, p<0.01\right)$. So the hypothesis 9 is proved to be true. An analysis using the same method is made on the regulating effect of leader-member commutation relation between leadership prestige and organizational identity of subordinate, but its regulating effect is found to be not significant, so it is not indicated in a list. The hypothesis 10 is not proved.

\section{Research conclusion and prospect}

\subsection{Research analysis and conclusion}

The outcome of this research indicates the following problems:

Firstly, this research makes hypotheses and proves the leadership prestige has a significant positive predictive effect on the leader trust and organizational identity of subordinate. When the leadership prestige of the managers is high, its subordinate will also present a high level of leadership trust and organizational identity. Boxun Zheng (1999) thought that in the Chinese cultural and organizational environment, there's a huge gap between the powers of leaders and subordinates. The leaders have an ability to allocate the organizational resources, while the subordinates tend to obtain some resources or meet their own demands with the help of the leaders. When the leaders have a high influence and prestige in the leadership mode and behavior, it will be easier for them to get the trust and favor from their subordinates, and it also increases the trust and identity of subordinates to the organization.

Secondly, this research makes further discussion about the mechanism of action of the leadership prestige on the attitude and behavior of subordinate. The research result proves that the leader trust and organizational identity have a complete mediation effect between the leadership prestige and the working satisfaction and working investment of subordinates. This result shows the subordinate will form its identity assessment to the leader under the influence of leadership prestige. The reason why the superior manager will have a prestige among the subordinates is that the emotional sense of belonging and resonance of the subordinates are induced after they develop a sense of trust for the superior and a sense of identity for the organization, which stimulates their working enthusiasm, increases the working satisfaction and produces significant positive effect on the working investment conditions.

Thirdly, the Chinese cultures and customs shall be adequately focused on in the research of leadership science in the Chinese cultural environment. This research shows the leadership and leader-member commutation relation will both intensify the positive relation between the leadership prestige and organizational identity of subordinate. It means the leadership prestige, as an abstractive influence, will have a positive effect on the mind and behavior of the subordinates and significantly increase their identity with the organization only if it is efficiently used by the managers with efficient management mode and management behavior, as well as through their efficient power application and good relation between the superior and subordinate. Furthermore, the research result also indicates the effect of leadership prestige on subordinate's trust for leader is not affected by the leadership and leader-member commutation relation.

\subsection{Research limitation and prospect}

There are also some imperfections in this research: (1) the demographic variables have not been added in the discussion about the relation of leadership prestige and each variable, which may produce higher coefficients related to the regression relation. In the future, we may conduct a further research on the correlation of leadership prestige and a deeper discussion about the 
influence mechanism of the leadership prestige on each of the main variables in organizational behavior. (2) Each variable, mainly provided by the respondents themselves, may have homological deviation; and the tested selection is unable to actually achieve the random sampling, which causes interference in the variable relation. Therefore in the future research, we shall make every effort to overcome the sampling problems easily posed in the social investigation method and use a vertical research design with large time span. In the investigation of antecedent variables and outcome variables, we shall retain a certain amount of time interval and make attempt to get the tested investigation samples in more regions and enterprises to enhance the stability and universality of the research conclusion.

\section{Acknowledgement}

Fund project: MOE (Ministry of Education in China) Youth Project of Humanities and Social Sciences (Project No. 10YJC630074); The Study on the Content Structure and Influence Mechanism of Leader Prestige; Introducing Talent Project of Guangdong University of Foreign Studies.

\section{References}

1. Avolio B J, Gardner W L, Walumbwa F O, et al. Unlocking the Mask a Look at the Process by Which Authentic Leaders Impact Follower Attitudes and Behaviors [J]. Leadership Quarterly, 2004, 15(6): 801-823.

2. Farh, J. L., Tsui, A. S., Xin, K. R., \& Cheng, B. S. The Influence of Relational Demography and Guanxi: The Chinese Case [J]. Organization Science, 1998, 9: 471-488.

3. Luthans F, Avolio B J. Authentic Leadership: A positive Developmental Approach. Positive Organizational Scholarship [M]. San Francisco: Barrett- Koehler, 2003.

4. Fan, J.L., Zheng, B.X. Paternalistic Leadership Organized by Chinese Organization: Analysis on A Cultural Opinion [J]. Research on Local Psychology, 2000, 13: 127-180.

5. Pan, X.F., Qin, Q.W. Measurement of Organizational Atmosphere in Middle School and Relevant Analysis on Psychological Health of Teachers [J]. Psychological Science, 2007, 30(4): 982-986.

6. Qian, S.H., Min, W.G. Brief Discussion on Flexible Management and Establishment of Modern Leadership Prestige [J]. Academic Exploration, 2002, 3.

7. Zheng, Q. Power and Prestige of Leader Cadres --Reflection on Leader Power and Prestige [J]. Journal of Hunan Industry Polytechnic 